\title{
Simulación numérica del comportamiento no-lineal de materiales utilizando el método sin malla de puntos finitos
}

\author{
Meshless numerical simulation of the non-linear behavior of a material \\ using the finite point method
}

\author{
Luis Pérez P. ${ }^{1} \quad$ Franco Perazzo M. ${ }^{1} \quad$ Carlos Spa C. ${ }^{2}$ \\ Recibido 11 de mayo de 2011, aceptado 9 de diciembre de 2011 \\ Received: May 11, 2011 Accepted: December 9, 2011
}

\begin{abstract}
RESUMEN
La formulación sin malla del método de puntos finitos permite aprovechar en toda su potencialidad la ventaja de este tipo de técnica numérica, habiéndose comprobado su buen funcionamiento para aplicaciones en los campos de la mecánica de fluidos, mecánica de sólidos, ciencia de materiales y más tarde en adaptividad y electromagnetismo.

En el presente trabajo se desarrolla una metodología numérica para aproximar el comportamiento no-lineal de un material mediante el método de puntos finitos, basada en la teoría de deformación total de Hencky, en conjunto con un enfoque elástico para aproximar la distribución de tensiones y de deformaciones. Esta aproximación introduce el concepto de propiedades efectivas del material, las cuales se obtienen en forma iterativa mediante un procedimiento de corrección aplicado sobre la curva experimental de tensióndeformación. Los ejemplos desarrollados demuestran el correcto comportamiento de la metodología utilizada, siendo una de sus principales ventajas la sencillez y facilidad de su implementación, puesto que no es necesaria la partición o subdivisión del dominio de solución.
\end{abstract}

Palabras claves: Métodos sin malla, método de puntos finitos, colocación puntual, análisis no-lineal, análisis inelástico.

\begin{abstract}
The use of fully meshless formulation of the finite point method allows taking advantage the benefit of this type of numerical technique for applications in the fields of fluid mechanics, solid mechanics, material science and later in adaptivity and electromagnetism.

In this work a meshless numerical method to approximate the non-linear behavior of a material using the finite point method, based on the theory of Hencky total strain and elastic approach to approximate the distribution of stresses and deformation, is developed. This approach introduces the concept of effective properties of the material which are obtained using a correction procedure applied to the stress-strain curve. The examples show the good behavior of the methodology that is used, being one of the main advantages the simplicity and the ease of implementation, since it is not necessary a partition or subdivision of the solution domain.
\end{abstract}

Keywords: Meshless methods, finite point method, point collocation, non-linear analysis, inelastic analysis.

\footnotetext{
1 Departamento de Ingeniería Mecánica, Universidad Técnica Federico Santa María, Av. España 1680, Valparaiso, Chile. E-mail: luis.perez@usm.cl; franco.perazzo@usm.cl

2 Departamento de Matemática. Universidad Técnica Federico Santa María. Av. Vicuña Mackenna 3939, Santiago, Chile. E-mail: carlos.spa@usm.cl
} 


\section{INTRODUCCIÓN}

Diversos trabajos de investigación desarrollados durante la última década permiten comprender el importante avance en la aplicación de un nuevo tipo de técnicas numéricas que, a diferencia de los métodos tradicionales como el de elementos finitos o volúmenes finitos, no requieren de una subdivisión del dominio o malla. Se ha denominado naturalmente a estas técnicas métodos sin malla o libres de malla. Estas comprenden un conjunto de métodos numéricos utilizados para resolver ecuaciones diferenciales parciales con condiciones de contorno, a partir de distribuciones de puntos regulares o irregulares. Mayores detalles acerca de estas técnicas y sus ventajas pueden encontrarse en $[1,16,17,19,20]$.

De entre los métodos sin malla más representativos, cabe la pena mencionar a: Generalized Finite Difference (GFD) [3], Diffuse Element (DE) [4], Element Free Galerkin (EFG) [5] y [6], Reproducing Kernel Particle Method (RKPM) [7] y [8], Hp Clouds Method [1], Partition of Unity (PU) [9-10], Finite Points Method (FPM), Método de Puntos Finitos [2, 11], Local Boundary Integral Equation (LBIE) [12-13], Meshless Local Petrov-Galerkin (MLPG) [14] y Point Interpolation Method (PIM) [15-16].

En el caso particular del Método de Puntos Finitos (MPF), propuesto por Oñate y otros [2,11], su primera utilización fue con el objeto de resolver problemas de transporte convectivo y de mecánica de fluidos. Posteriormente su aplicación se extendió a problemas de transporte difusivo advectivo [21] y de flujo incompresible [22]. En el contexto de la mecánica de sólidos, el MPF ha sido aplicado en problemas de elasticidad [23-25], dinámica de sólidos [26] y recientemente en refinamiento adaptivo [27-28].

La idea de utilizar soluciones elásticas para aproximar el comportamiento no-lineal de materiales tiene su origen en el trabajo pionero de Neuber [29]. Utilizando esta técnica, el investigador desarrolló una teoría para la concentración del esfuerzo en cuerpos prismáticos que tienen este tipo de comportamiento constitutivo. Con posterioridad, se utilizó un análisis elástico en conjunto con el Método de Elementos Finitos (MEF) para estimar cargas límites [30], técnica que fue utilizada y mejorada en el trabajo de Seshadri [31], dando origen al método denominado Generalized Local Stress and Strain (GLOSS). Siguiendo esta línea de investigación, en [32] se desarrolló un método para el análisis inelástico de estanques de presión, utilizando un número finito de franjas, llamado Variable Material Properties (VPM). Posteriormente, mediante un enfoque pseudoelástico, se utilizó el MEF con la idea de analizar el comportamiento no-lineal de un material [33].

Recientemente, en el contexto de los métodos sin malla basados en una formulación variacional del problema, se utilizó el EFG para realizar un análisis pseudoelástico de la no-linealidad del material [34] y posteriormente en [35] se empleó el mismo concepto pero utilizando el PIM. En ambos casos, si bien es cierto el cálculo de la función de forma no depende de la disponibilidad de una subdivisión del dominio, la resolución del problema mediante su forma débil supone contar con una malla de fondo o malla de integración.

En este trabajo se presenta una metodología totalmente libre de malla, basada en el Método de Puntos Finitos, para el análisis de no-linealidad del material. Para determinar la distribución de esfuerzos y deformaciones se utiliza la teoría de deformación total de Hencky y un enfoque elástico. Dicha aproximación introduce el concepto de propiedades efectivas del material, las cuales son consideradas como variables de campo. Estos parámetros se obtienen en forma iterativa mediante un procedimiento de corrección aplicado sobre la curva experimental de tensión uniaxial del material.

Para verificar la validez de la metodología propuesta se comparan diversos ejemplos en 2D con los resultados que se obtienen mediante el uso de un software comercial basado en el MEF.

\section{EL MÉTODO DE PUNTOS FINITOS}

Se presentan a continuación las principales expresiones de la técnica de interpolación por mínimos cuadrados utilizadas en el MPF. Un estudio más extenso de esta técnica, sus propiedades y características respecto de las aproximaciones utilizadas en otros métodos sin malla, ha sido desarrollado en [24]. 
Sea $\Omega_{I}$ el subdominio de interpolación, llamado usualmente nube de puntos en los métodos sin malla, de una función $u(x)$ y $s(j)$ con $j=1,2, \ldots$, $n$ una colección de $n$ puntos con coordenadas $x_{j} \in \Omega_{I}$, donde la coordenada $x_{j}$ es una coordenada local, adimensional, y centrada en el nodo estrella de la nube. Mayores detalles sobre este cambio de coordenadas se encuentran en $[2,11,23,28]$. La función incógnita $u$ puede ser aproximada en el interior $\Omega_{I}$ por:

$$
u(x) \cong \hat{u}(x)=\sum_{k=1}^{m} p_{k}(x) \alpha_{k}=p(x)^{T} \alpha
$$

Donde $\alpha$ es un vector de coeficientes de ponderación constantes y $p(x)$ un vector denominado base de interpolación, el cual contiene típicamente monomios que aseguran una base completa en el espacio de coordenadas. Como ejemplo para el caso 2D se tiene:

$$
\begin{gathered}
p=[1, x, y]^{T} \text { para } m=3 \\
p=\left[1, x, y, x^{2}, x y, y^{2}\right]^{\mathrm{T}} \text { para } m=6
\end{gathered}
$$

La función incógnita $u(x)$ puede ser evaluada en los $n$ puntos pertenecientes a la nube $\Omega_{I}$, obteniendo:

$$
u^{h}=\left\{\begin{array}{c}
u_{1}^{h} \\
u_{2}^{h} \\
\vdots \\
u_{n}^{h}
\end{array}\right\} \cong\left\{\begin{array}{c}
\hat{u}_{1} \\
\hat{u}_{2} \\
\vdots \\
\hat{u}_{n}
\end{array}\right\} \cong\left\{\begin{array}{c}
p_{1}^{T} \\
p_{2}^{T} \\
\vdots \\
p_{n}^{T}
\end{array}\right\} \alpha=C \alpha
$$

Donde los valores $u_{j}^{h}=u\left(x_{j}\right)$ son las incógnitas, pero los valores buscados $u$ en el punto $j, \hat{u}_{j}=\hat{u}\left(x_{j}\right)$ son los valores aproximados y $p_{j}=p\left(x_{j}\right)$.

En una aproximación mediante el MEF, el número de puntos en el subdominio se escoge de forma que $m=n$. En este caso $C$ es una matriz cuadrada y el procedimiento conduce a las funciones de forma estándar del MEF [36].

Si $m>n$ la aproximación utilizada no se puede ajustar a todos los valores de $u_{j}^{h}$. Este problema puede ser resuelto determinando los valores de $\hat{u}$ que minimicen la suma de la distancia al cuadrado o error en cada punto, ponderado por una función $w(x)$, es decir:

$$
\begin{aligned}
& J=\sum_{j=1}^{n} w\left(x_{j}\right)\left(u_{j}^{h}-\hat{u}\left(x_{j}\right)\right)^{2} \\
& =\sum_{j=1}^{n} w\left(x_{j}\right)\left(u_{j}^{h}-p_{j}^{T} \alpha\right)^{2}
\end{aligned}
$$

Esto corresponde a una aproximación por mínimos cuadrados ponderados Weighted Least Square (WLS). Se debe notar que si en (5) se elige $w(x)=1$ se obtiene la aproximación por mínimos cuadrados estándar Least Square (LSQ).

La función de ponderación $w(x)$ utilizada en el MPF corresponde a la función de Gauss normalizada. Otras funciones utilizadas en el contexto de los métodos sin malla pueden ser consultadas en [2, 11, 24, 37].

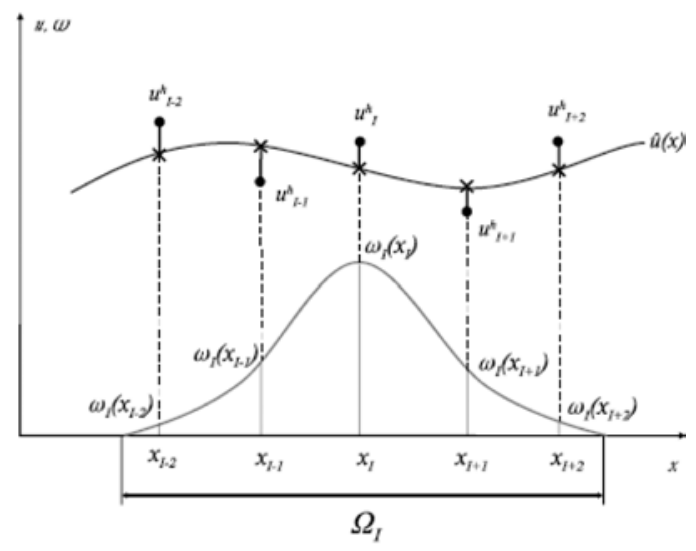

Figura 1. Aproximación por mínimos cuadrados ponderados (WLS).

Tomando mínimo respecto de $\alpha$ en Ec. (5), se obtiene:

$$
\alpha=\bar{C}^{-1} u^{h}, \bar{C}^{-1}=A^{-1} B
$$

$$
\begin{aligned}
& A=\sum_{j=1}^{n} w\left(x_{j}\right) p\left(x_{j}\right) p^{T}\left(x_{j}\right) \\
& B=\left[w\left(x_{1}\right) p\left(x_{1}\right), w\left(x_{2}\right) p\left(x_{2}\right), \ldots, w\left(x_{n}\right) p\left(x_{n}\right)\right]
\end{aligned}
$$

La aproximación final del MPF se obtiene reemplazando $\alpha$ de Ec. (6) en Ec. (1) obteniendo: 


$$
\hat{u}(x)=p^{T} \bar{C}^{-1} u^{h}=\Phi^{T} u^{h}=\sum_{j=1}^{n} \varphi_{j}^{I} u_{j}^{h},
$$

Donde

$$
\varphi_{j}^{I}(x)=\sum_{l=1}^{m} p_{l}(x) \bar{C}_{l j}^{-1}=p^{T}(x) \bar{C}^{-1}
$$

son las funciones de forma.

Se debe notar que debido al carácter de mínimos cuadrados de la aproximación, $u\left(x_{j}\right) \cong \hat{u}\left(x_{j}\right) \neq u_{j}^{h}$, es decir, los valores locales de la función aproximada, no coinciden con los valores nodales de la función incógnita, ver Figura 1. De todas formas $\hat{u}$ es una aproximación válida, con la cual se busca satisfacer la ecuación diferencial y condiciones de contorno, siendo $u_{j}^{h}$ simplemente parámetros desconocidos.

La aproximación mediante mínimos cuadrados ponderada descrita anteriormente depende en gran medida de la forma y la manera de aplicar la función de ponderación $w(x)$. La forma más simple consiste en definirla como una función fija en cada uno de los subdominios $\Omega_{I}[2,11,37]$, entonces:

$$
\begin{array}{ll}
w_{I}\left(x_{I}\right)=1 & \\
w_{I}(x) \neq 0 & x \in \Omega_{I} \\
w_{I}(x)=0 & x \notin \Omega_{I}
\end{array}
$$

A partir de la minimización de (5), se obtiene:

$$
J_{I}=\sum_{j=1}^{n} w_{I}\left(x_{j}\right)\left(u_{j}^{h}-\hat{u}\left(x_{j}\right)\right)^{2} \text { mínimo }
$$

Siendo las expresiones para las matrices $A$ y $B$ coincidentes con Ec. (7) cuando

$$
w\left(x_{j}\right)=w_{I}\left(x_{j}\right)
$$

De acuerdo con Ec. (1), la función aproximada $\hat{u}(x)$ se define para cada subdominio de interpolación $\Omega_{I}$, por lo que la aproximación para un punto $I$ que pertenezca a dos nubes $\Omega_{I}$ y $\Omega_{K}$, respectivamente, no tiene un único valor $\varphi_{j}^{I} \neq \varphi_{j}^{K}$. En el MPF esta disyuntiva se resuelve utilizando un procedimiento de colocación puntual.

\section{ESFUERZO-DEFORMACIÓN BASADO EN LA TEORÍA DE DEFORMACIÓN TOTAL}

De acuerdo a [32], la relación esfuerzo-deformación puede expresarse como:

$$
\varepsilon_{i j}=f\left(\sigma_{i j}\right)
$$

Donde $\varepsilon_{i j}$ y $\sigma_{i j}$ son el tensor de deformación total y el tensor de esfuerzos, respectivamente, además $\varepsilon_{i j}$ puede ser descompuesto en una componente elástica y una plástica.

$$
\varepsilon_{i j}=\varepsilon_{i j}^{e}+\varepsilon_{i j}^{p}
$$

Usando la ley de Hooke para materiales isótropos, el tensor de deformaciones elásticas se relaciona con el tensor de esfuerzos por:

$$
\varepsilon_{i j}^{e}=\frac{1+v}{E} \sigma_{i j}-\frac{v}{E} \delta_{i j} \sigma_{k k}
$$

siendo $E$ y $v$ el módulo de Young y el coeficiente de Poisson, respectivamente.

Por otro lado, la teoría de la deformación total de Hencky [38] establece una relación entre la componente plástica del tensor de deformación y la parte desviadora del tensor de esfuerzos, dada por:

$$
\varepsilon_{i j}^{p}=\psi S_{i j}
$$

Donde

$$
S_{i j}=\sigma_{i j}-\frac{1}{3} \delta_{i j} \sigma_{k k}
$$

es la parte desviadora del tensor de esfuerzos y $\psi \mathrm{es}$ una función escalar que se obtiene de la siguiente forma:

$$
\psi=\frac{3}{2} \frac{\varepsilon_{e q}^{p}}{\sigma_{e q}}
$$

Utilizando notación tensorial y el convenio de suma sobre el índice repetido, la definición en forma explícita de la deformación plástica equivalente $\left(\varepsilon^{p}{ }_{e q}\right)$ y el esfuerzo equivalente $\left(\sigma_{e q}\right)$ es [32]: 


$$
\varepsilon_{e q}^{p}=\sqrt{\frac{2}{3} \varepsilon_{i j}^{p} \varepsilon_{i j}^{p}} \quad y \quad \sigma_{e q}=\sqrt{\frac{3}{2} S_{i j} S_{i j}}
$$

Reemplazando la Ec. (14) y (15) en (13) se obtiene:

$$
\varepsilon_{i j}=\left(\frac{1+v}{E}+\psi\right) \sigma_{i j}-\left(\frac{v}{E}+\frac{1}{3} \psi\right) \delta_{i j} \sigma_{k k}
$$

Esta expresión puede reescribirse como:

$$
\varepsilon_{i j}=\left(\frac{1+v_{e f f}}{E_{e f f}}\right) \sigma_{i j}-\left(\frac{v_{e f f}}{E_{e f f}}\right) \delta_{i j} \sigma_{k k}
$$

Donde $E_{\text {eff }}$ es el módulo de Young efectivo y $v_{\text {eff }}$ es el coeficiente de Poisson efectivo, los cuales son función de $E, v$ y $\psi$.

Para obtener los parámetros efectivos del material se debe comparar la Ec. (19) con Ec. (20), con lo cual:

$$
\begin{aligned}
& E_{\text {eff }}=\left(\frac{1}{E}+\frac{2}{3} \psi\right)^{-1} \\
& v_{\text {eff }}=E_{\text {eff }}\left(\frac{v}{E}+\frac{1}{3} \psi\right)
\end{aligned}
$$

\section{FORMA DISCRETA DE LAS ECUACIONES DE EQUILIBRIO}

Considérese el siguiente sistema de ecuaciones diferenciales, las cuales gobiernan el equilibrio de un sólido y cuyas fuerzas de inercia son despreciables:

$$
L^{T} \sigma+\rho b=0 \quad \forall x \in \Omega-\Gamma
$$

y condiciones de contorno

$$
\begin{gathered}
N^{T} \sigma=\bar{t} \quad \forall x \in \Gamma_{t} \\
u=\bar{u} \quad \forall x \in \Gamma_{u}
\end{gathered}
$$

Con $\Omega$ el dominio de solución del problema y $\Gamma$ su contorno $\left(\Gamma=\Gamma_{t}+\Gamma_{u}\right)$. Para un caso $2 \mathrm{D}, L$ es un operador que define la ecuación diferencial, $N$ la matriz que contiene los cosenos directores en la dirección normal al contorno, $\sigma\left(\tau_{x y}=\tau_{y x}\right)$ es el vector de esfuerzos, $u$ es el vector de desplazamientos, $\rho, b, \bar{t}$ y $\bar{u}$ son la densidad volumétrica del sólido, las fuerzas de cuerpo, tracciones externas y desplazamientos prescritos respectivamente. Utilizando notación ingenieril, estas variables se pueden agrupar en los siguientes arreglos:

$$
\begin{gathered}
\boldsymbol{L}:=\left[\begin{array}{cc}
\frac{\partial}{\partial x} & 0 \\
0 & \frac{\partial}{\partial y} \\
\frac{\partial}{\partial y} & \frac{\partial}{\partial x}
\end{array}\right], \quad \boldsymbol{N}:=\left[\begin{array}{cc}
n_{x} & 0 \\
0 & n_{y} \\
n_{y} & n_{x}
\end{array}\right] \\
\sigma:=\left[\begin{array}{c}
\sigma_{x} \\
\sigma_{y} \\
\tau_{x y}
\end{array}\right], \quad \boldsymbol{u}:=\left[\begin{array}{l}
u \\
v
\end{array}\right] \\
\boldsymbol{b}:=\left[\begin{array}{c}
b_{x} \\
b_{y}
\end{array}\right], \overline{\boldsymbol{t}}:=\left[\begin{array}{c}
\overline{\boldsymbol{t}}_{x} \\
\overline{\boldsymbol{t}}_{y}
\end{array}\right], \overline{\boldsymbol{u}}:=\left[\begin{array}{c}
\bar{u} \\
\bar{v}
\end{array}\right]
\end{gathered}
$$

Para obtener un sistema equivalente en términos de los desplazamientos, se debe usar la relación esfuerzo-deformación de la siguiente forma:

$$
\begin{aligned}
& \sigma=D \varepsilon \\
& \varepsilon=L u
\end{aligned}
$$

Donde $D$ representa la matriz simétrica constitutiva, la cual, de acuerdo a Ec. (20), viene dada por:

$$
D=\left[\begin{array}{ccc}
d_{11} & d_{12} & 0 \\
d_{21} & d_{22} & 0 \\
0 & 0 & d_{33}
\end{array}\right]
$$

donde sus coeficientes quedan definidos como:

$$
\begin{aligned}
& d_{11}=d_{22}=\frac{\bar{E}}{1-\bar{v}^{2}} \\
& d_{12}=d_{21}=\bar{v}^{2} d_{11} \\
& d_{33}=\frac{\bar{E}}{2(1+\bar{v})}
\end{aligned}
$$

$\bar{E} \begin{cases}E_{\text {eff }} & \text { tensión plana } \\ E_{\text {eff }} /\left(1-v_{\text {eff }}\right) & \text { deformación plana }\end{cases}$

$\bar{v} \begin{cases}v_{\text {eff }} & \text { tensión plana } \\ v_{\text {eff }} /\left(1-v_{\text {eff }}\right) & \text { deformación plana }\end{cases}$ 
y $E_{\text {eff, }}, v_{\text {eff }}$ se obtienen mediante Ec. (21) y Ec. (22) respectivamente.

Reemplazando la Ec. (26) en (23), (24) y (25), utilizando el procedimiento de colocación puntual y la aproximación del MPF definida en Ec. (8), se obtiene el siguiente sistema de ecuaciones discretas:

$$
\begin{aligned}
& {\left[L D L^{T} \boldsymbol{\Phi}+(L \Phi D) L^{T} \Phi\right] u^{h}=-\rho b \quad \forall \mathrm{x} \in \Omega-\Gamma} \\
& {\left[N^{T} D L \Phi\right] u^{h}=\bar{t} \quad \forall x \in \Phi_{t}} \\
& {[\Phi] u^{h}=\bar{u} \quad \forall x \in \Gamma_{u}}
\end{aligned}
$$

En un problema elástico lineal, la expresión ( $L \Phi D)$ entre paréntesis en Ec. (28) se anula, debido a que la matriz $D$ es constante.

Para cada nodo $I$ del dominio, el sistema de Ecuaciones (28) se puede expresar como:

$$
K_{I} u^{h}=f_{I} \quad I=1, \ldots ., N
$$

Donde la matriz $K_{I}$ está compuesta por $n$ submatrices $K_{I}^{i}$, definidas de la siguiente forma:

$$
\begin{aligned}
& \text { •si } I \in \Omega-\Gamma \\
& \mathrm{K}_{I}^{\mathrm{i}}=\left\{\begin{array}{l}
{\left[\mathrm{K}_{I}^{\mathrm{i}}\right]_{11}=d_{11} \varphi_{I, x x}^{i}+d_{33} \varphi_{I, y y}^{i}+\left(d_{11, x} \varphi_{I, x}^{i}+d_{33, y} \varphi_{I, y}^{i}\right)} \\
{\left[\mathrm{K}_{I}^{\mathrm{i}}\right]_{12}=\varphi_{I, x y}^{i}\left(d_{12}+d_{33}\right)+\left(d_{12, x} \varphi_{I, y}^{i}+d_{33, y} \varphi_{I, x}^{i}\right)} \\
{\left[\mathrm{K}_{I}^{\mathrm{i}}\right]_{21}=\varphi_{I, x y}^{i}\left(d_{21}+d_{33}\right)+\left(d_{21, y} \varphi_{I, x}^{i}+d_{33, x} \varphi_{I, y}^{i}\right)} \\
{\left[\mathrm{K}_{I}^{\mathrm{i}}\right]_{22}=d_{22} \varphi_{I, y y}^{i}+d_{33} \varphi_{I, x x}^{i}+\left(d_{22, y} \varphi_{I, y}^{i}+d_{33, x} \varphi_{I, x}^{i}\right)}
\end{array} \quad, \mathrm{f}_{I}=-\rho \mathrm{b}\right. \\
& \text { •si } I \in \Gamma_{t} \\
& \mathrm{~K}_{I}^{\mathrm{i}}=\left\{\begin{array}{c}
{\left[\mathrm{K}_{I}^{\mathrm{i}}\right]_{11}=d_{11} \varphi_{I, x}^{i} n_{x}+d_{33} \varphi_{I, y}^{i} n_{y}} \\
{\left[\mathrm{~K}_{I}^{\mathrm{i}}\right]_{12}=d_{12} \varphi_{I, y}^{i} n_{x}+d_{33} \varphi_{I, x}^{i} n_{y}} \\
{\left[\mathrm{~K}_{I}^{\mathrm{i}}\right]_{21}=d_{33} \varphi_{I, y}^{i} n_{x}+d_{12} \varphi_{I, x}^{i} n_{y}} \\
{\left[\mathrm{~K}_{I}^{\mathrm{i}}\right]_{22}=d_{33} \varphi_{I, x}^{i} n_{x}+d_{22} \varphi_{I, y}^{i} n_{y}}
\end{array}, \mathrm{f}_{I}=\overline{\mathrm{t}}\right. \\
& \text {-si } I \in \Gamma_{u} \\
& \mathrm{~K}_{I}^{\mathrm{i}}=\left\{\begin{array}{c}
{\left[\mathrm{K}_{I}^{\mathrm{i}}\right]_{11}=\left[\Phi_{I}^{i}\right]_{11}} \\
{\left[\mathrm{~K}_{I}^{\mathrm{i}}\right]_{12}=0} \\
{\left[\mathrm{~K}_{I}^{\mathrm{i}}\right]_{21}=0} \\
{\left[\mathrm{~K}_{I}^{\mathrm{i}}\right]_{22}=\left[\mathrm{K}_{I}^{i}\right]_{11}}
\end{array}, \mathrm{f}_{I}=\overline{\mathrm{u}}\right.
\end{aligned}
$$

\section{DETERMINACIÓN DE LOS PARÁMETROS EFECTIVOS DEL MATERIAL}

En este trabajo se utiliza el método de proyección vertical propuesto en [32] para determinar los parámetros efectivos del material,

$$
E_{\text {eff }} \mathrm{y} v_{\text {eff }}
$$

Considerando un punto genérico a, se obtiene en primer lugar una solución elástica, ver Figura 2. Esto definirá el estado equivalente inicial de esfuerzo-deformación en ese punto, $E_{0},\left(\sigma_{e q}, \varepsilon_{e q}\right)$. Luego, el valor de deformación $\varepsilon_{e q_{a}}$ se fija y se proyecta sobre la curva de tensión del material, lo que permite calcular el estado de esfuerzo $\sigma_{e q}$, correspondiente al nuevo punto a' proyectado en la curva. Finalmente, el valor actualizado de $E_{\text {eff }}$ se obtiene mediante la razón entre el esfuerzo y deformación equivalente, es decir,

$$
E_{e f f}=\frac{\sigma_{e q_{\mathrm{a}}}}{\varepsilon_{e q_{\mathrm{a}}}}
$$

El correspondiente valor actualizado de $v_{\text {eff }}$ se debe calcular usando la Ec. (22). Este procedimiento se debe repetir hasta que el estado de esfuerzodeformación de cada punto coincida con la curva uniaxial de esfuerzo del material.

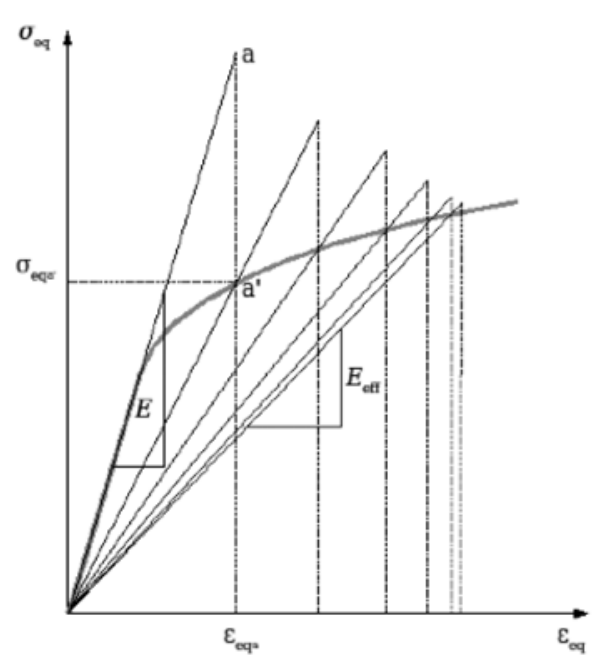

Figura 2. Método de proyección vertical. 
Para un material con una ley de endurecimiento descrita por el modelo de Ramberg-Osgood [39] se tiene:

$$
\frac{\varepsilon_{\mathrm{eq}}}{\varepsilon_{0}}=\frac{\sigma_{e q}}{\sigma_{0}}+\alpha_{R O}\left(\frac{\sigma_{e q}}{\sigma_{0}}\right)^{n_{R O}}
$$

Donde el esfuerzo equivalente $E_{e q_{a}}$, usado en Ec. (30) y Figura 2 se obtiene mediante un solver no-lineal basado en el método de Newton [35].

Los valores de $E_{\text {eff }} \mathrm{y} v_{\text {eff }}$ de acuerdo a Ec. (30) y (22) respectivamente, se pueden expresar de la siguiente forma:

$$
\begin{gathered}
E_{\text {eff }}=\left(\frac{1}{E_{0}}+\alpha_{R O} \frac{\varepsilon_{\text {eq }}}{\varepsilon_{0}}\left(\frac{\sigma_{e q}}{\sigma_{0}}\right)^{n_{R O}-1}\right)^{-1} \\
v_{\text {eff }}=E_{e f f}\left(\frac{v_{0}}{E_{0}}+\alpha_{R O} \frac{1}{2} \frac{\varepsilon_{0}}{\sigma_{0}}\left(\frac{\sigma_{e q}}{\sigma_{0}}\right)^{n_{R O}-1}\right)
\end{gathered}
$$

Donde $\varepsilon_{0}=\sigma_{0} / E_{0}$ es la deformación correspondiente al inicio de la fluencia $\sigma_{0}, \alpha_{R O}$ es un parámetro de ajuste y $n_{R O}$ es el exponente de endurecimiento. Tanto el parámetro de ajuste como el exponente de endurecimiento se deben asignar al comienzo del análisis.

\section{EJEMPLOS NUMÉRICOS}

Los siguientes ejemplos numéricos permiten corroborar la correcta implementación del método propuesto, utilizándose los siguientes parámetros de acuerdo a la aproximación del MPF; una base de interpolación cuadrática $(m=6)$ y nubes de $n=9$ puntos. Por otro lado, la tolerancia en el método de proyección vertical se ha fijado en 0.001 .

\section{Placa rectangular sujeta a tensión constante}

En este problema se aplica la metodología propuesta en este trabajo para análisis de una placa rectangular sujeta a tensión constante $\sigma_{c}$. Se ha considerado una condición de tensión plana, utilizándose un material no-lineal descrito por el modelo de Ramberg-Osgood [39], mediante la siguiente fórmula:

$$
\varepsilon_{e q}=\underbrace{\frac{\sigma_{e q}}{E}}_{\varepsilon_{e q}^{e}}+\underbrace{\frac{\sigma_{0} \alpha_{R O}}{E}\left(\frac{\sigma_{e q}}{\sigma_{0}}\right)^{n_{R O}}}_{\varepsilon_{e q}^{p}}
$$

la cual se utiliza para obtener los valores de la deformación equivalente $\varepsilon_{e q}$ y deformación plástica equivalente $\varepsilon_{e q}^{p}$ correspondientes a la tensión prescrita.

Las magnitudes de $\varepsilon_{e q}$ y $\varepsilon_{e q}^{p}$ resultantes de la simulación mediante el MPF se comparan (ver Tabla 2) con los valores obtenidos previamente usando la Ecuación (34). Nótese que producto de las condiciones de carga se cumple $\sigma_{e q}=\sigma_{c}$.

La geometría del dominio y condiciones de contorno se entregan en la Figura 3. La discretización utilizada se compone de 36 puntos. Sobre la parte superior de la placa se prescribe la tensión $\sigma_{c}$. Los parámetros del modelo de Ramberg-Osgood se muestran en la Tabla 1.

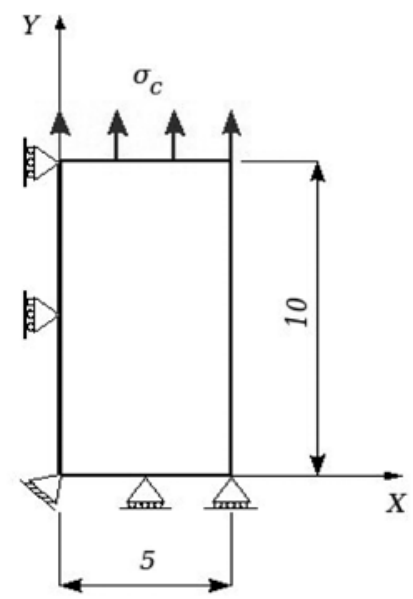

Figura 3. Geometría y condiciones de contorno para una placa sujeta a tensión constante (dimensiones en $[\mathrm{cm}]$ ).

Tabla 1. Parámetros del material, placa rectangular sujeta a tensión constante.

\begin{tabular}{|l|l|l|}
\hline Módulo de elasticidad & $E$ & $2 \cdot 10^{5} \mathrm{MPa}$ \\
\hline Relación de Poisson & $v$ & 0.3 \\
\hline Esfuerzo de fluencia & $\sigma_{0}$ & $200 \mathrm{MPa}$ \\
\hline Parámetro de Ajuste & $\alpha_{\mathrm{RO}}$ & $2 \cdot 10^{-6}$ \\
\hline Exponente de endurecimiento & $n_{R O}$ & 5 \\
\hline
\end{tabular}


La Tabla 2 resume los principales resultados que se obtienen utilizando el MPF y la presente metodología. En esta tabla se incluye además el número de iteraciones $N_{\text {iter }}$ empleadas en la resolución de cada caso estudiado.

Las Figuras 4, 5 y 6 muestran gráficamente el funcionamiento de la técnica propuesta y del método de proyección. También es posible apreciar el número de iteraciones $N_{\text {iter }}$.

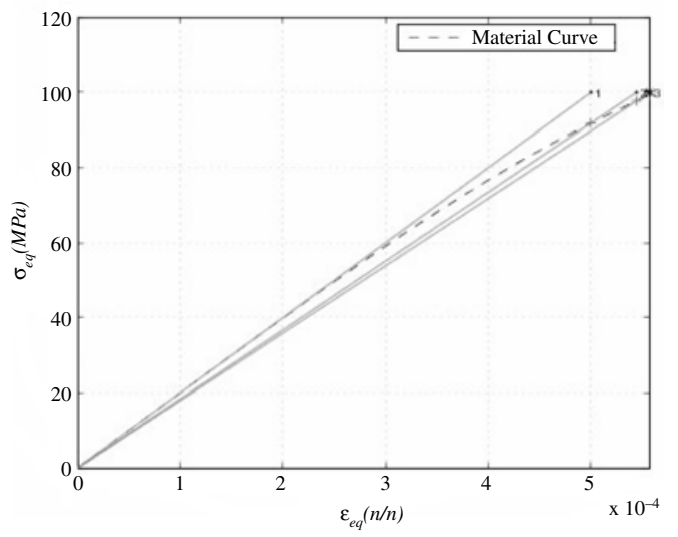

Figura 4. Gráfico de convergencia de un punto, placa rectangular sujeta a tensión constante $\sigma_{c}=100 M P a$.

Este problema permite comprobar y observar gráficamente el funcionamiento de la estrategia presentada en este trabajo.

A partir de la Tabla 2 se concluye que esta técnica es capaz de reproducir correctamente el comportamiento de un material no-lineal descrito por el modelo de Ramberg-Osgood.

Se observa en las Figuras 4, 5 y 6 que el número de iteraciones aumenta en forma proporcional a la

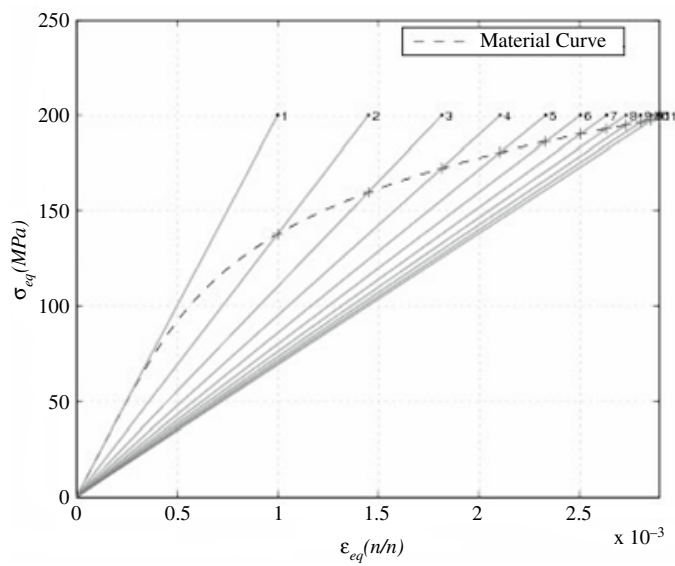

Figura 5. Gráfico de convergencia de un punto, placa rectangular sujeta a tensión constante $\sigma_{c}=200 \mathrm{MPa}$.

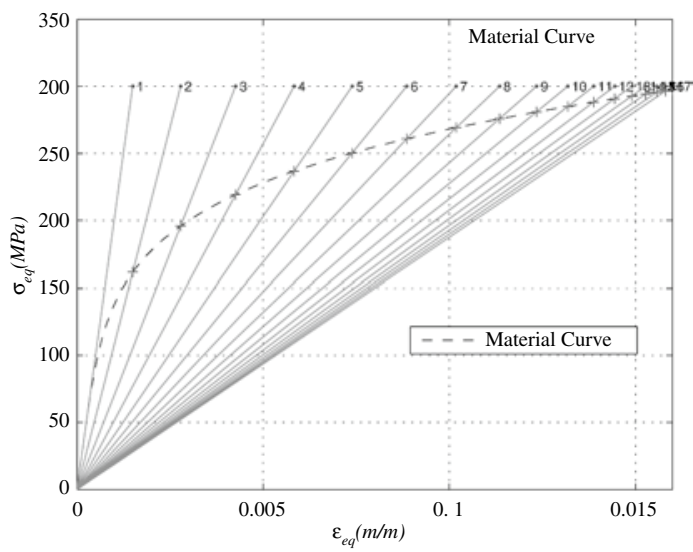

Figura 6. Gráfico de convergencia de un punto, placa rectangular sujeta a tensión constante $\sigma_{c}=300 \mathrm{MPa}$.

tensión prescrita. Este comportamiento se debe a que son necesarias una mayor cantidad de correcciones para reproducir el fenómeno no-lineal. Nótese la diferencia de estados equivalentes de tensióndeformación entre la Figuras 4 y 6.

Tabla 2. Principales resultados placa rectangular sujeta a tensión constante.

\begin{tabular}{|c|c|c|c|c|c|c|c|}
\hline$\sigma_{C[M P a]}$ & $\varepsilon_{e q(R . O)}$ & $\varepsilon^{p}{ }_{e q(R . O)}$ & $\varepsilon_{e q}$ & $\varepsilon^{p}$ & $E_{\text {eff }} / E$ & $v_{\text {eff }} / v$ & $N_{\text {iter }}$ \\
\hline 50 & 0.000250 & 0 & 0.000250 & 0 & 1 & 1 & 1 \\
\hline 100 & 0.000563 & 0.000063 & 0.000557 & 0.000050 & 0.8976 & 1.0682 & 3 \\
\hline 150 & 0.001225 & 0.000475 & 0.001201 & 0.000451 & 0.6243 & 1.2504 & 7 \\
\hline 200 & 0.003000 & 0.002000 & 0.002894 & 0.001894 & 0.3454 & 1.4363 & 11 \\
\hline 250 & 0.007350 & 0.006104 & 0.007089 & 0.005839 & 0.1763 & 1.5491 & 15 \\
\hline 300 & 0.016688 & 0.015188 & 0.015989 & 0.014489 & 0.0938 & 1.6041 & 17 \\
\hline
\end{tabular}




\section{Cilindro de pared gruesa con presión interna} Un segundo ejemplo consiste en el análisis no-lineal de un cilindro de pared gruesa de radio interior $R_{i n t}$ y radio exterior $R_{\text {ext }}$, con una presión interna $P i$. Este ejemplo ha sido utilizado ampliamente en el contexto de la simulación numérica del comportamiento nolineal de materiales mediante el uso de soluciones elásticas [32-35]. Se ha considerado una condición de deformación plana y un material no-lineal cuyos parámetros para el modelo de Ramberg-Osgood se muestran en la Tabla 3.

Tabla 3. Parámetros del material, cilindro de pared gruesa con presión interna.

\begin{tabular}{|l|l|l|}
\hline Módulo de elasticidad & $E$ & $2 \cdot 10^{5} \mathrm{MPa}$ \\
\hline Relación de Poisson & $v$ & 0.3 \\
\hline Esfuerzo de fluencia & $\sigma_{0}$ & $200 \mathrm{MPa}$ \\
\hline Parámetro de ajuste & $\alpha_{R O}$ & $3 / 7$ \\
\hline $\begin{array}{l}\text { Exponente de } \\
\text { endurecimiento }\end{array}$ & $n_{R O}$ & 5 \\
\hline
\end{tabular}

La geometría del dominio y las condiciones de contorno se muestran en la Figura 7. Se considera una relación $R_{\text {ext }} / R_{\text {int }}=5$. Se han analizado tres casos de presión interna: $P_{i} / \sigma_{0}=1.0,1.5$ y 2.0 .

La discretización utilizada se compone de 1.056 puntos.

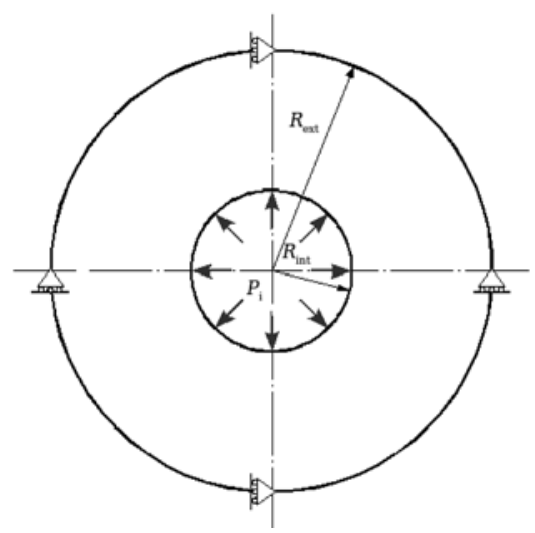

Figura 7. Geometría del cilindro de pared gruesa con presión interna.

Para este ejemplo, los resultados que se obtienen con la presente metodología se han comparado con los que entrega un software comercial del MEF.

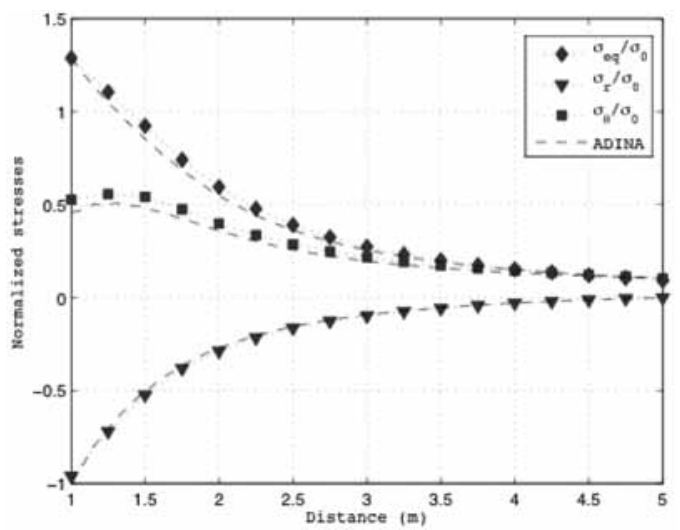

a)

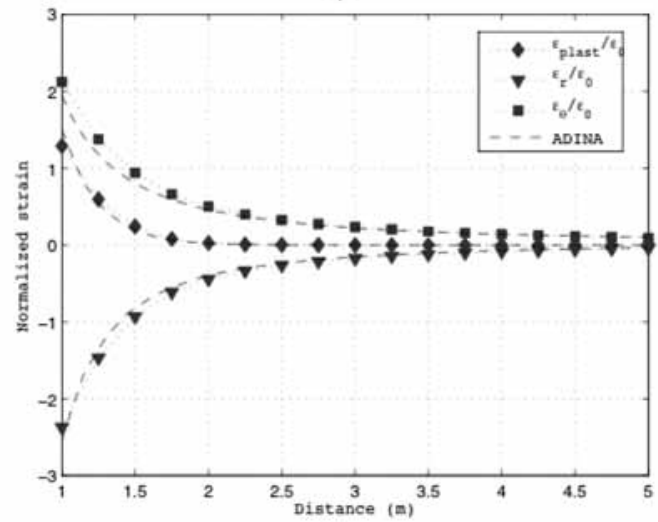

b)

Figura 8. Distribución normalizada de a) tensiones, b) deformaciones, cilindro de pared gruesa con presión interna, $P_{i} / \sigma_{0}=1$.

Se observa de las Figuras 8, 9 y 10 un aumento de la zona plástica conforme se incrementa la relación de $P_{0} / \sigma_{0}$, nótese además como estas variables normalizadas permiten representar de buena forma el avance del fenómeno no-lineal global.

Se observa además en la Figura 10 una leve diferencia en la distribución de las deformaciones normalizadas, esto se debe al elevado nivel de carga, lo que implica un avanzado estado no-lineal, el cual dificulta la convergencia de la solución.

Se puede apreciar en las Figuras 8, 9 y 10 que los resultados numéricos se ajustan en buena forma a los que entrega la modelación mediante el software comercial del MEF. Se concluye entonces que esta técnica es capaz de reproducir el comportamiento de un material no-lineal descrito por el modelo de Ramberg-Osgood. 


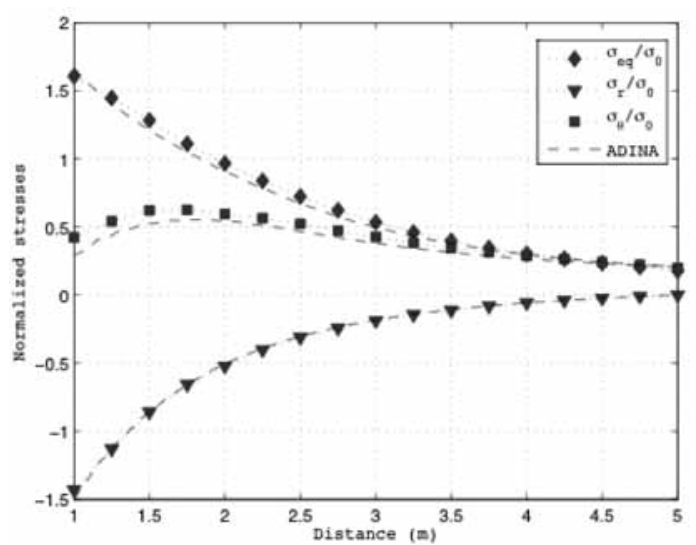

a)

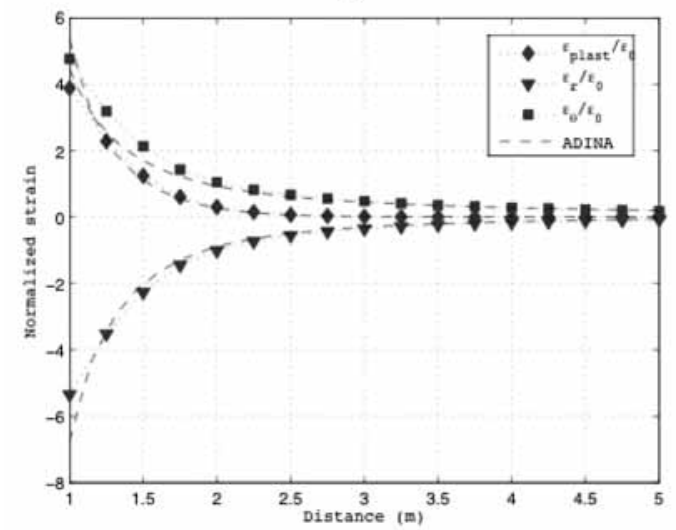

b)

Figura 9. Distribución normalizada de a) tensiones, b) deformaciones, cilindro de pared gruesa con presión interna, $P_{i} / \sigma_{0}=1.5$.

Placa rectangular con carga distribuida central El objetivo de este ejemplo es mostrar la efectividad de la técnica propuesta cuando se utiliza conjuntamente con un procedimiento para estabilizar la solución numérica. El uso de un esquema de colocación puntual en el MPF implica, como toda técnica numérica basada en una formulación fuerte, que las ecuaciones de equilibrio del problema solamente se imponen en el interior del dominio. Lo anterior, sumado a la falta de simetría de las nubes en el contorno, suele afectar la exactitud de los resultados cuando no se utiliza un número suficiente de puntos de colocación [23]. La técnica de estabilización utilizada en el presente ejemplo corresponde al procedimiento de Cálculo Finitesimal (FIC), la cual se ha utilizado con éxito en la aplicación del MPF en mecánica de sólidos [25-28, 40]. Se resuelve numéricamente

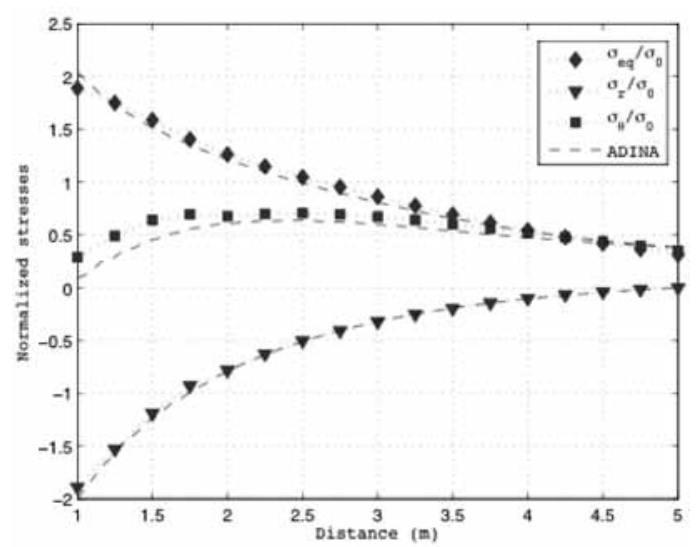

a)

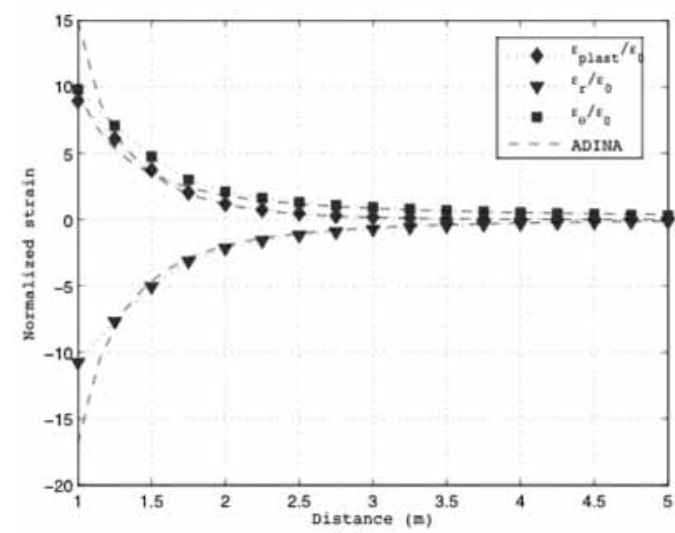

b)

Figura 10. Distribución normalizada de a) tensiones, b) deformaciones, cilindro de pared gruesa con presión interna, $P_{i} / \sigma_{0}=2$.

el problema mediante el MPF sin estabilizar y el MPF estabilizado, comparándose posteriormente los resultados obtenidos en cada simulación.

Se considera una condición de tensión plana y un material no-lineal dado por el modelo RambergOsgood cuyos parámetros se muestran en la Tabla 4.

Tabla 4. Parámetros del material, placa rectangular con carga distribuida central.

\begin{tabular}{|l|l|l|}
\hline Módulo de elasticidad & $E$ & $2 \cdot 10^{5} \mathrm{Mpa}$ \\
\hline Relación de Poisson & $v$ & 0.3 \\
\hline Esfuerzo de fluencia & $\sigma_{0}$ & $200 \mathrm{Mpa}$ \\
\hline Parámetro de ajuste & $\alpha_{R O}$ & $2 \cdot 10^{-6}$ \\
\hline $\begin{array}{l}\text { Exponente de } \\
\text { endurecimiento }\end{array}$ & $n_{R O}$ & 5 \\
\hline
\end{tabular}


La geometría del dominio y las condiciones de contorno se muestran en la Figura 11; se considera una carga distribuida de $w=250[\mathrm{~N} / \mathrm{mm}]$.

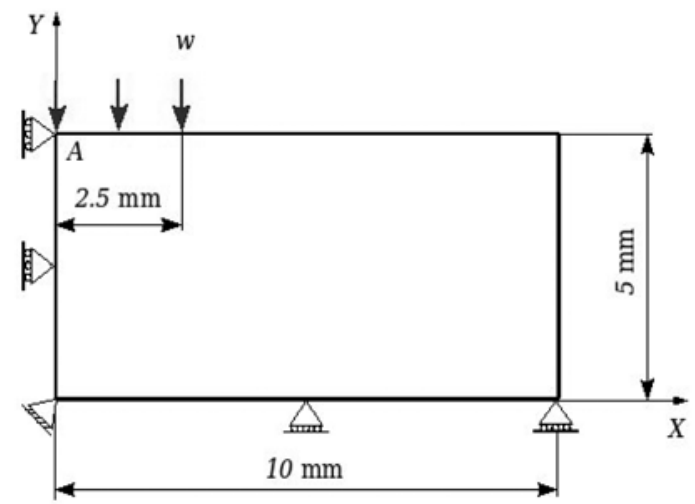

Figura 11. Geometría de la placa rectangular con carga distribuida central.

La Figura 12 muestra una comparación de la distribución normalizada de tensión equivalente $\sigma_{e q}$ y deformación plástica equivalente $\varepsilon^{p}{ }_{e q}$, en la parte superior de la placa, para la resolución numérica mediante MPF sin estabilizar y estabilizado. En la Figura 13 se muestra una comparación entre la geometría deformada de la placa, para el caso con y sin estabilización, cuando se utilizan isolíneas de colores para representar el desplazamiento total.

Las Figuras 12 y 13 permiten observar en este caso las inestabilidades que introduce la condición de Neumann en el procedimiento de colocación puntal, haciéndose totalmente necesario el uso de una técnica de estabilización, en este caso FIC.

\section{Cilindro de pared gruesa con presión interna y tensión cortante}

En este problema se aplica la presente técnica al análisis no-lineal de un cilindro de pared gruesa de radio interior $R_{i}$ y radio exterior $R_{\text {ext }}$, sujeto a una presión interna $P_{i}$ y una tensión cortante $\tau_{i}$.

Los resultados de este ejemplo se analizan en los trabajos de Jahed [32] y Sethuraman \& Reddy [34], para la simulación numérica del comportamiento no-lineal de materiales empleando aproximaciones elásticas, pero en el contexto de un método sin malla con aproximación variacional.

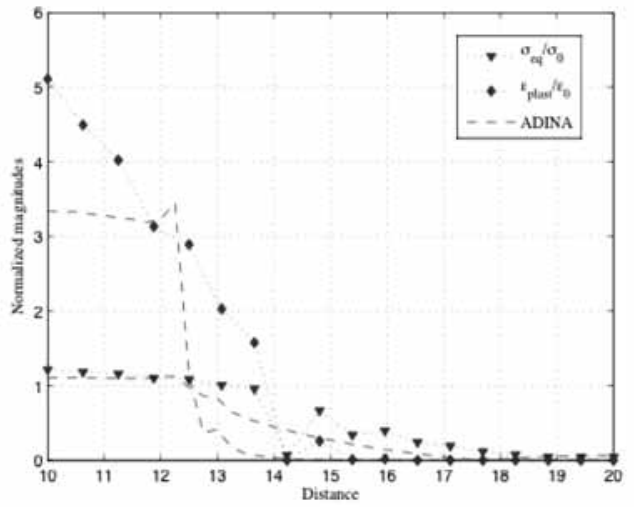

a)

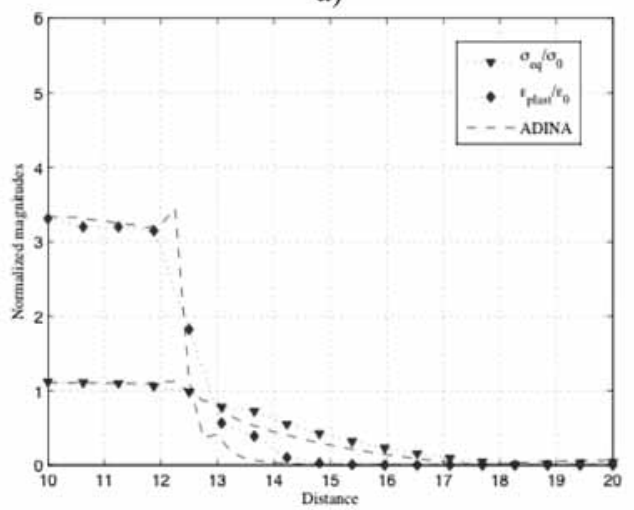

b)

Figura 12. Distribución normalizada de tensión equivalente $\sigma_{e q}$ y deformación plástica equivalente $\varepsilon_{e q}^{p}$, placa rectangular con carga distribuida central. a) MPF sin estabilizar, b) MPF estabilizado.

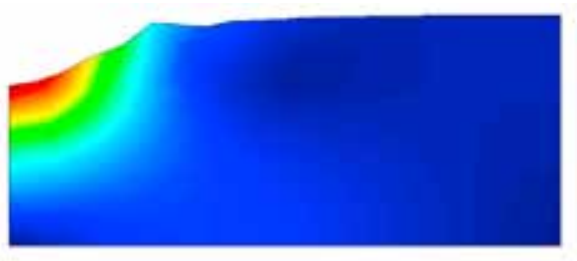

a)

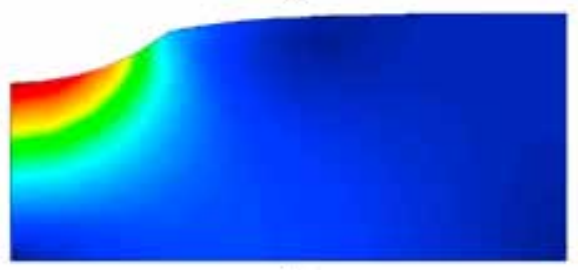

b)

Figura 13. Geometría deformada, placa rectangular con carga distribuida central. a) MPF sin estabilizar, b) MPF estabilizado. 
En la resolución de este ejemplo se consideran condiciones de deformación plana y tensión plana, con un material cuya relación constitutiva viene dada por el modelo de Ramberg-Osgood (ver Tabla 5).

Tabla 5. Parámetros del material, cilindro de pared gruesa con presión interna y tensión cortante.

\begin{tabular}{|l|l|l|}
\hline Módulo de elasticidad & $E$ & $2 \cdot 10^{5} \mathrm{Mpa}$ \\
\hline Relación de Poisson & $v$ & 0.3 \\
\hline Esfuerzo de fluencia & $\sigma_{0}$ & $200 \mathrm{Mpa}$ \\
\hline Parámetro de ajuste & $\alpha_{R O}$ & $3 / 7$ \\
\hline $\begin{array}{l}\text { Exponente de } \\
\text { endurecimiento }\end{array}$ & $n_{R O}$ & 5 \\
\hline
\end{tabular}

La geometría del dominio y las condiciones de contorno se muestran en la Figura 14; se considera una relación Rext/Rint $=5$. La discretización utilizada se compone de 812 puntos. Los valores para la presión interna y la tensión cortante vienen dados por:

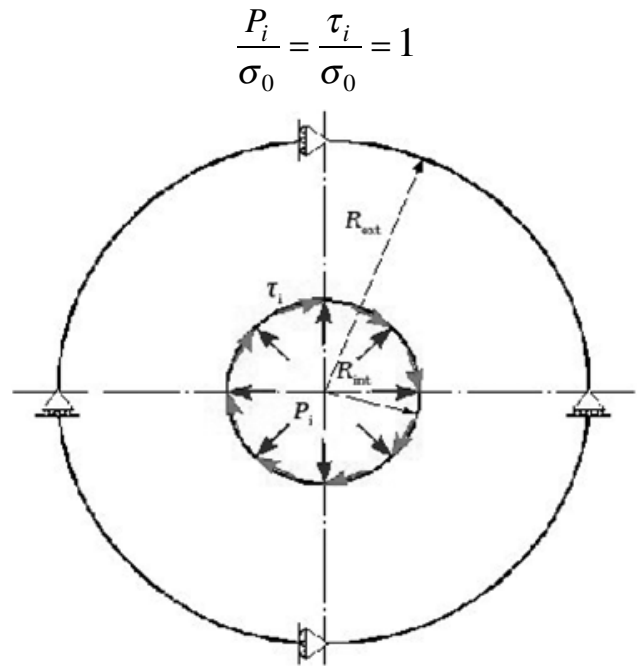

Figura 14. Geometría del cilindro de pared gruesa con presión interna y tensión cortante.

Los resultados que se obtienen con la presente metodología se han comparado con los que se entregan en el trabajo de Sethuraman \& Reddy [34]. Las Figuras 15 y 16 muestran respectivamente la distribución normalizada de tensiones y deformaciones para las dos condiciones estudiadas.

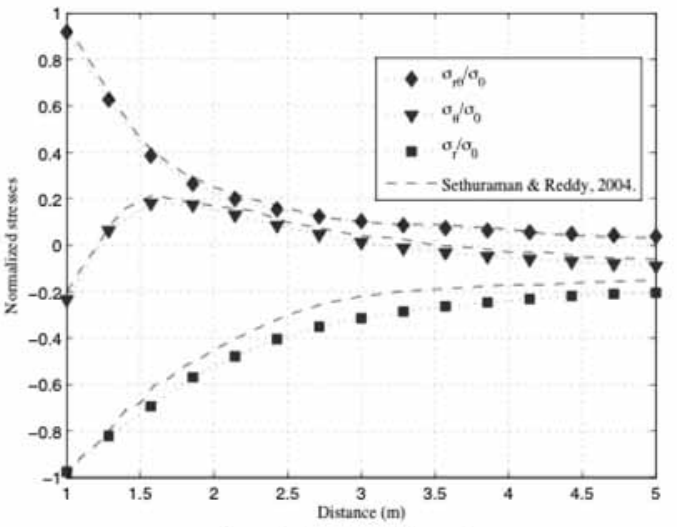

a) deformación plana

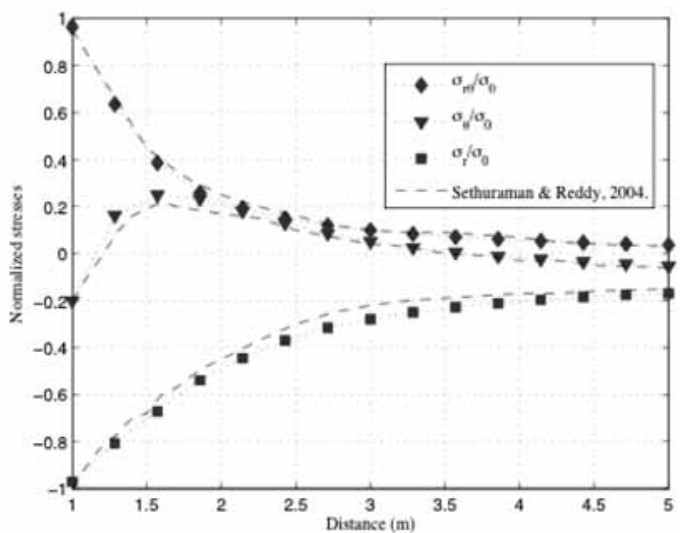

b) tensión plana

Figura 15. Distribución normalizada de tensiones, cilindro de pared gruesa con presión interna y tensión cortante.

Tal como se observa en las Figuras 15 y 16, el MPF reproduce de buena forma el efecto combinado de la presión interna y tensión cortante. Además, los resultados numéricos que se obtienen se ajustan en buena forma con los entregados en el trabajo de Sethuraman \& Reddy [34]. Estos resultados permiten otra vez corroborar que, con la utilización de una aproximación numérica sin malla basada en el MPF, se puede reproducir correctamente el comportamiento no-lineal de un material descrito por el modelo de Ramberg-Osgood.

\section{CONCLUSIONES}

En este trabajo se ha presentado el desarrollo y la implementación de una metodología sin malla, basada en el Método de Puntos Finitos, para el análisis de la no-linealidad de un material. 


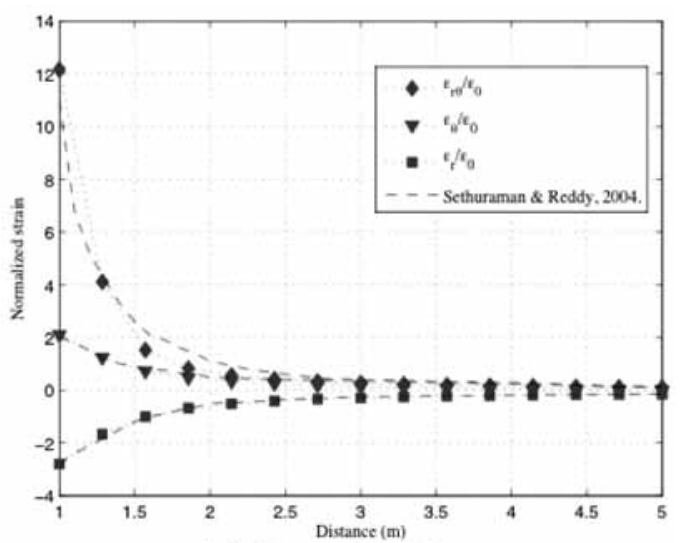

a) deformación plana

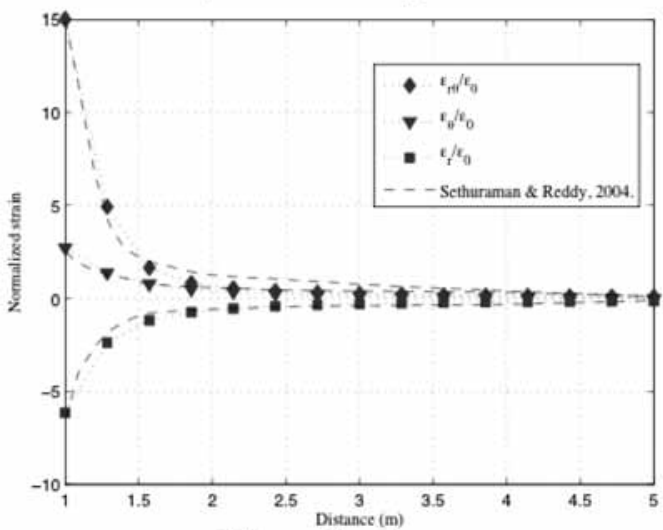

b) tensión plana

Figura 16. Distribución normalizada de deformaciones, cilindro de pared gruesa con presión interna y tensión cortante.

Para determinar la distribución de esfuerzos y deformaciones se ha utilizado la teoría de deformación total de Hencky y un enfoque elástico. Se ha utilizado el concepto de propiedades efectivas del material, las cuales son consideradas como variables de campo, para obtener una solución al presente problema no-lineal.

Los ejemplos numéricos desarrollados permiten demostrar la capacidad de esta técnica para simular el comportamiento no-lineal de un material descrito por el modelo de Ramberg-Osgood. Comparada con otras técnicas, por ejemplo teoría incremental y el método de Newton-Raphson, el uso del MPF demuestra ser una técnica sencilla de implementar debido a que no es necesario subdividir el dominio de solución para realizar integración alguna. Cabe mencionar que esta técnica ha sido desarrollada mediante una formulación fuerte, forma poco abordada en la literatura existente relativa al uso de este tipo de aproximación no-lineal.

\section{AGRADECIMIENTOS}

Los autores agradecen el aporte financiero de CONICYT a través del proyecto FONDECYT 11100253 , así como también los proyectos UTFSM 251150 y 251156.

\section{REFERENCIAS}

[1] Y.T. Gu. "Meshfree methods and their comparisons". International Journal of Computational Methods. Vol. 2, pp. 477515. 2005.

[2] E. Oñate, S. Idelsohn, O.C. Zienkiewicz and R. Taylor. "A finite point methods in computational mechanics, application to convective transport and fluid flow". International Journal for Numerical Methods in Engineering. Vol. 39, pp. 3839-3866. 1996.

[3] T. Liszka and J. Orkisz. "The finite difference method at arbitrary irregular grids and its application in applied mechanics". Computers and Structures. Vol. 11, pp. 83-95. 1980.

[4] B. Nayroles, G. Touzot and P. Villon. "Generalizing the fem: Diffuse approximation and diffuse elements". Computational Mechanics. Vol. 10, pp. 307-318. 1992.

[5] T. Belytschko, Y. Lu and L. Gu. "Element free galerkin methods". International Journal for Numerical Methods in Engineering. Vol. 37, pp. 229-256. 1994.

[6] Y. Lu, T. Belytschko and L. Gu. "A new implementaion of the element free Galerkin method". Computer Methods in Applied Mechanics and Engineering. Vol. 113, pp. 397-414. 1994.

[7] W.K. Liu, S. Jun and Y.F. Zhang. "Reproducing kernel particle methods". International Journal for Numerical Method in Engineering. Vol. 20, pp. 1081-1106. 1995.

[8] W.K. Liu, Y. Chen, S. Jun, J.S. Chen, T. Belytschko, C. Pan, R.A. Uras and C.T. Chang. "Overview and applications of the reproducing kernel particle method". Archives of Comput. Meth. in Engng. Vol. 3, pp. 3-80. 1996. 
[9] I. Babuska and J.M. Melenk. "The partition of unity finite element method". Institute for Physics, Science, and Technology, University of Maryland, Maryland. Technique Report. 1995.

[10] I. Babuska and J.M. Melenk. "The partition of unity finite element method: basic theory and applications". Computer Methods in Applied Mechanics and Engineering. Vol. 139, pp. 289-314. 1996.

[11] E. Oñate, S. Idelsohn, O. Zienkiewics, R. Taylor and C. Sacco. "A stabilized finite point method for analysis of fluid mechanics problems". Computer Methods in Applied Mechanics and Engineering. Vol. 139, pp. 315-346. 1996.

[12] T. Zhu, J. Zhang and S.N. Atluri. "A local boundary integral equation (lbie) method in computational mechanics and a meshless discretization approach". Computational Mechanics. Vol. 21, pp. 223-235. 1998.

[13] T. Zhu, J. Zhang and S.N. Atluri. "A meshless local boundary integral equation (lbie) method for solving nonlinear problems". Computational Mechanics. Vol. 2, pp. 174186. 1998.

[14] SN. Atluri and T. Zhu. "A new meshless local petrov-galerkin (mlpg) approach in computational mechanics". Computational Mechanics. Vol. 22, pp. 117-127. 1998.

[15] G.R. Liu and Y.T. Gu. "A point interpolation method for two-dimensional solids". International Journal for Numerical Methods in Engineering.Vol. 50, pp. 937-951. 2001.

[16] G.R. Liu. "Mesh Free Methods: Moving Beyond the Finite Element Method". CRC press. USA. 2002.

[17] C. Duarte. "A review of some meshless methods to solve partial differential equations". PhD thesis. 1995.

[18] M. Chao. "Introducción a los métodos numéricos sin malla: Fundamentación y desarrollo de formulaciones de mínimos cuadrados ponderados". $\mathrm{PhD}$ thesis. España. 1997.

[19] Y. Chen, J. Lee and A. Eskandarian. "Meshless methods in solids mechanics". Springer. New York. USA. 2006.

[20] S. Li and W.K. Liu. "Meshfree particle methods”. Springer. Berlín, Alemania. 2004.
[21] E. Oñate and S. Idelsohn. "A mesh free finite point method for advective-diffusive transport and fluid flow problems". Computational Mechanics. Vol. 21, pp. 283-292. 1998.

[22] E. Oñate, C. Sacco and S. Idelsohn. "A finite point method for incompressible flow problems". Computer Visual Science. Vol. 3, pp. 67-75. 2000.

[23] E. Oñate, F. Perazzo and J. Miquel. "A finite point method for elasticity problems". Computer and Structures. Vol. 79, pp. 21512163. 2001.

[24] F. Perazzo. "Una metodología numérica sin malla para la resolución de las ecuaciones de elasticidad mediante el método de puntos finitos". Tesis para optar al grado de doctor. Universitat Politécnica de Cataluña. Barcelona, España. 2002.

[25] F. Perazzo, S. Oller, J. Miquel and E. Oñate. "Avances en el método de puntos finitos para la mecánica de sólidos". Revista Internacional de Métodos Numéricos en Ingeniería. Vol. 22, pp. 153-168. 2006.

[26] F. Perazzo, J. Miquel and E. Oñate. "El método de puntos finitos para problemas de la dinámica de sólidos". Revista Internacional de Métodos Numéricos en Ingeniería. Vol. 20, pp. 235-246. 2004.

[27] F. Perazzo, R. Lohner and L. Pérez-Pozo. "Adaptive methodology for meshless finite point method". Advances in Engineering Software. Vol. 39, pp. 156-166. 2008.

[28] A. Angulo, L. Pérez-Pozo and F. Perazzo. "A posteriori error estimator and an adaptive technique in meshless Finite Points Method". Engineering Analysis With Boundary Elements. Vol. 33, pp. 1322-1338. 2009.

[29] H. Neuber. "Theory of stress concentration for shear strained prismatical bodies with arbitrary nonlinear stress-strain law". ASME Applied Mechanics. Vol. 28, pp. 554-560. 1961.

[30] A.K. Dhalla and G.L. Jones. "Asme code classification of pipe stresses: a simplified procedure". International Journal of Pressure Vessel Piping. Vol. 26, pp. 145-166. 1986.

[31] R. Seshadri. "The generalized local stress-strain (gloss) analysis theory and applications". Journal of Pressure Vessel Technology ASME. Vol. 113, pp. 219-227. 1991. 
[32] H. Jahed, R. Sethuraman and R.N. Dubey. "A variable material property approach for solving elastic-plastic problems". International Journal of Pressure Vessel Piping. Vol. 71, pp. 285-291. 1997.

[33] V. Desikan and R. Sethuraman. "Analysis of material nonlinear problems using pseudoelastic finite element method". Journal of Pressure Vessel Technology ASME. Vol. 122, pp. 457-461. 2000.

[34] R. Sethuraman and S. Reddy. "Pseudo-elastic analysis of material non-linear problems using element free galerkin method". Journal of the Chinese Institute of Engineers. Vol. 27, pp. 505-516. 2004.

[35] K.Y. Dai, G.R. Liu, X. Han and Y. Li. "Inelastic analysis of $2 \mathrm{~d}$ solids using a weak. form rpim based on deformation theory". Computer methods in applied mechanics and engineering. Vol. 195, pp. 4179-4193. 2005.
[36] O. Zienkiewicz and R. Taylor. "El método de los elementos finitos". Centro Internacional de Métodos Numéricos en Ingeniería. Vol. 1. Barcelona, España. 2000.

[37] R.L. Taylor, S. Idelsohn, O.C. Zienkiewicz and E. Oñate. "Moving least square approximations for solution of differential equations". CIMNE Research Report No 74. 1995.

[38] H. Hencky. "Zur theorie plastischer deformationen und der hierdurch im material hervorgerufenen nebenspannungen". Proceedings of the First International Congress on Applied Mechanics. Delft, Holanda. 1924.

[39] W. Ramberg and WR. Osgood. "Description on stress-strain curves by three parameters". National Advisory Committee for Aeronautics (NACA). 1943.

[40] E. Oñate. "Possibilities of finite calculus in computational mechanics". International Journal for Numerical Methods in Engineering. Vol. 60, pp. 255-281. 2004. 